\title{
The molecular recognition theory applied to bispecific antibodies ... .
}

To the editor - In a previous issue of Nature Medicine, J. Edwin Blalock outlined the potential importance of the molecular recognition theory (MRT) to our understanding of the structure-function relationship of proteins and peptides ${ }^{1}$. In the same issue, Baranyi et al. ${ }^{2}$ describe a new structural motif in proteins, the anti-
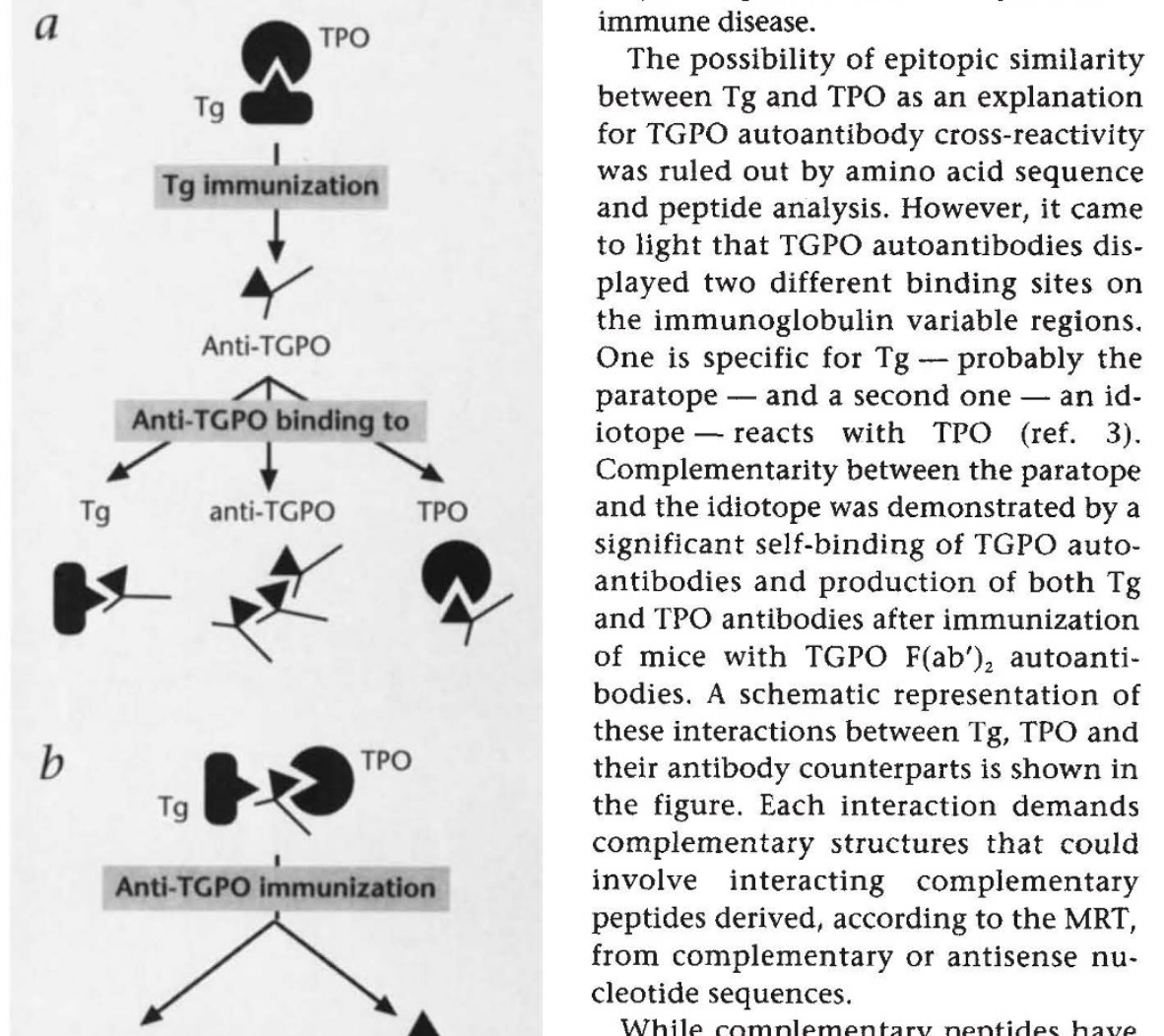

While complementary peptides have been independently associated with a variety of interacting peptides, so far they have not been implicated in interacting autoantigens and their corresponding antibodies. Our observation that TGPO antibodies may simultaneously bind $\mathrm{Tg}$ and TPO on separate interactive structures fits rather well with the proposed AHB theory. The paratope of the TGPO autoantibodies would bind to the major hormonogenic site of human $\mathrm{Tg}$, and this site, which should obviously interact with TPO to form thyroid hormones, would be complementary to both TPO and the paratope of the TGPO autoantibodies. Alternatively, an antisense idiotope complementary to the paratope would account for the binding of TPO to TGPO autoantibodies.

It would seem worthwhile to examine antibodies directed against complementary structures of interacting antigens, such as hormones and cognate receptors, for the presence of antisense sequences. This may improve our understanding of the autoimmune mechanisms leading to diseases implicating interacting autoantigens.

Jean Ruf \& Pierre Carayon

Unité 38 de l'Institut National

de la Santé et de la Recherche Médicale

and Laboratoire de Biochimie

Endocrinienne et Métabolique

Faculté de Médecine

F-13385 Marseille Cedex 5, France

\section{... but not to protein folding?}

To the editor - Blalock ${ }^{1}$ discusses the study of Baranyi et al. ${ }^{2}$ concerning antisense homology boxes (AHB) within protein molecules in which AHBs consist of amphiphilic peptides and corresponding antisense peptides separated by approximately 50 amino acids. In both articles there is speculation that AHBs may represent intra- and intermolecular recognition sites in proteins.

To investigate this possibility, we have analysed several proteins of known crystal structure. Thirty-four proteins were taken from the representative (nonhomologous) set of structures in the
Protein Data Bank $k^{4}$ : $\mathrm{AVH}, 1 \mathrm{BAB}, 1 \mathrm{DNK}$, 1END, 1FAS, 1FCS, 1FDD, 1FXI, 1GKY, 1MUP, 1OMP, 1OSA, 1PAF, 1PAZ, 1PHB, $1 \mathrm{RND}, 1 \mathrm{SBP}, 2 \mathrm{AAA}, 2 \mathrm{CPL}, 2 \mathrm{HAD}, 2 \mathrm{LIV}$, 2PIA, 2RN2, 2SN3, 3ADK, 3CD4, 3DFR, 4FXN, 4GCR, 4SBV, 8CAN, 9RNT, 1L92, 1TIE. Within this set there are, using the original criteria of Baranyi et al. (tenamino acid-wide frame and eight or more amino acids required to be antisense), only ten antisense homology boxes in nine proteins $(1 \mathrm{AVH}, 1 \mathrm{FXI}$, $1 \mathrm{GKY}, 1 \mathrm{PHB}, 2 \mathrm{AAA}, 2 \mathrm{PIA}, 4 \mathrm{GCR}, 8 \mathrm{ACN}$ and 1L92), and in none of these do the sense and antisense fragments interact 